\title{
Norois
}

Environnement, aménagement, société

\section{Conclusion : Vers une «technologie» de la gouvernance territoriale! Plaidoyer pour un programme de recherche sur les instruments et dispositifs de la gouvernance des territoires}

Eduardo Chia, André Torre et Hélène Rey-Valette

\section{(2) OpenEdition}

Journals

Édition électronique

URL : http://journals.openedition.org/norois/2603

DOI : $10.4000 /$ norois. 2603

ISBN : 978-2-7535-1557-4

ISSN : $1760-8546$

Éditeur

Presses universitaires de Rennes

\section{Édition imprimée}

Date de publication : 15 décembre 2008

Pagination : 167-177

ISBN : 978-2-7535-0805-7

ISSN : 0029-182X

\section{Référence électronique}

Eduardo Chia, André Torre et Hélène Rey-Valette, «Conclusion : Vers une «technologie» de la gouvernance territoriale! Plaidoyer pour un programme de recherche sur les instruments et dispositifs de la gouvernance des territoires », Norois [En ligne], 209 | 2008/4, mis en ligne le 01 mars 2009, consulté le 22 avril 2019. URL : http://journals.openedition.org/norois/2603 ; DOI : 10.4000/ norois. 2603 


\title{
Conclusion
}

\author{
VERS UNE « TECHNOLOGIE » DE LA GOUVERNANCE TERRITORIALE! \\ Plaidoyer POUR UN PROGRAMME DE RECHERCHE SUR LES INSTRUMENTS \\ ET DISPOSITIFS DE LA GOUVERNANCE DES TERRITOIRES
}

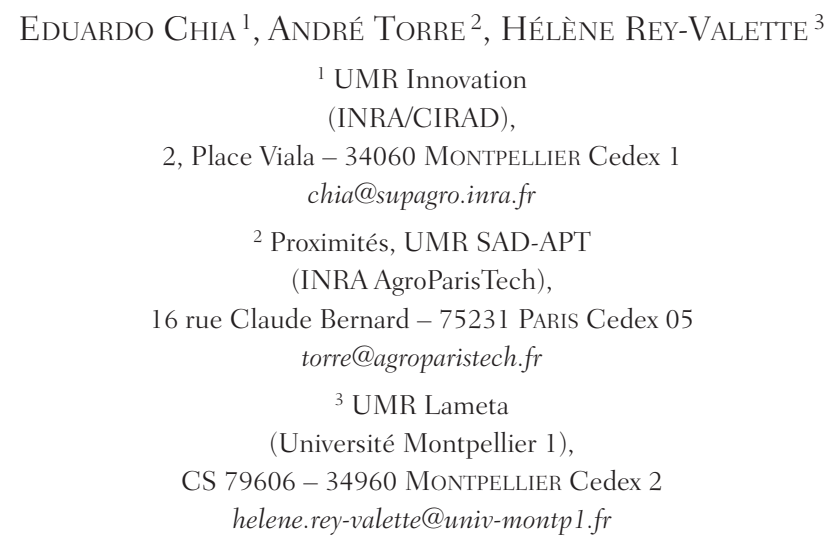

Le titre de ce numéro spécial «Dispositifs et outils de gouvernance territoriale » témoigne de l'intérêt de la participation de la géographie aux débats et aux controverses sur la gouvernance. Il s'agit en particulier de contribuer à préciser la notion de gouvernance territoriale. Comment participe-t-elle aux dynamiques territoriales? Quel rôle joue-t-elle dans les processus de développement des territoires? Comment les acteurs s'approprient ou élaborent-ils des instruments ou des dispositifs de coordination?

Les papiers présentés illustrent la grande diversité des situations et enjeux relatifs à la notion de gouvernance territoriale et montrent l'intérêt des approches pluridisciplinaires pour l'aborder. Cette diversité des disciplines constitue en effet un atout, à un double titre : sur le plan méthodologique, par la pluralité des approches et des modes d'accompagnement, et sur le plan de la capitalisation des connaissances, par le «stock » d'expérimentations contrastées tant du point de vue des situations que des problématiques abordées. En conclusion, nous allons nous intéresser à deux aspects qui nous paraissent importants pour clarifier les processus et les résultats de la gouvernance territoriale, à savoir les outils et dispositifs et les situations de gouvernance. L'écriture à trois mains permet d'associer deux points de vue, celui des «sciences de gestion » et de celui de l'économie non-standard. Après une brève synthèse de la façon dont les recherches en sciences sociales abordent la gouvernance, nous nous intéresserons au processus de gouvernance territoriale, en en révélant les facettes coopératives et conflictuelles, avant de montrer l’intérêt épistémologique des sciences de gestion pour étudier les dispositifs de gouvernance. 


\section{La construction d'une problématique de recherche sur la gouvernance : brève synthèse bibliographique}

La revue des définitions de la notion de gouvernance (Rey-Valette et al., 2008) témoigne du caractère polysémique et flou de cette notion, qui présente du coup l'avantage de permettre « des réceptions et usages différenciés grâce à du bricolage ", selon les termes de R. Pasquier et al. (2007). Ces auteurs appréhendent la gouvernance comme une grammaire, au sens « d'un ensemble de règles et de styles rendant possible la conduite d'une action publique » dans un contexte de différentiation (et d'autonomisation) de plus en plus poussée de la société (Le Gales, 1995) et de multiplication des parties prenantes. Elle est également souvent présentée comme un gouvernement du compromis (Taiclet, 2007), ou comme un « processus de coordination multi niveaux et multipolaires », dans un contexte polycentrique et fortement asymétrique (Gaudin, 1999). En continuité des innovations institutionnelles engendrées par la décentralisation et la contractualisation (Gaudin, 2002), les acteurs sont amenés à expérimenter de nouvelles formes d'action publique et de participation aux décisions, glissant, comme l'évoque F. Ost (2004), de la pyramide au réseau. Les dispositifs de gouvernance visent alors à faciliter la participation de porteurs d'intérêts de plus en plus diversifiés (notamment par l'association entre acteurs publics et privés) à des processus de décisions qui se révèlent sans cesse plus fragmentés et distribués, mais aussi incertains et mouvants.

La gouvernance apparaît ainsi comme un point focal, cristallisant de nombreux apports récents sur les questions d'interaction, d'action collective, «d'empowerment » et d'apprentissage... Toutefois, elle ne constitue un objet spécifique que pour quelques disciplines; la science politique, la sociologie, les sciences de gestion, l'économie institutionnelle, voire l'épistémologie pour les relations entre science et décision, les sciences de l'information pour le rôle des objets médiateurs et l'économie de la connaissance pour les processus d'apprentissage. Même si le plus souvent les travaux sont interdisciplinaires, on note que ce sont les approches de science politiques qui placent la gouvernance au cœur de leur problématique (Lahaye, 1999; Capelier et Simoulin, 2003; Hufy et al., 2007). Pour les autres, on identifie le plus souvent quelques thématiques clés, qui caractérisent des objets ou des problématiques particulières : l'expertise et l'action publique (Lascoumes, 2005), l'intérêt général (Denis, 2008), la gouvernance participative (Heurgon et Landrieu, 2001), les droits de propriété (Keefer, 2004 ; Platteau, 2003), la gouvernance communautaire (Clark et al., 2007), la gouvernance en matière de développement (Meisel et Ould Aoudia, 2007), les politiques publiques (Baslé, 2000 ; Larrue 2000), la gouvernance située en relation avec la problématique de la proximité (Bertrand, 2004 ; Zaoual, 2005; Talbot, 2006 ; Torre et Zuindeau, 2006) et notamment, à propos des mesures agro-environnementales, la question des dispositifs d'engagement volontaire (auto réglementation, chartre, accords volontaires...) (Salles, 2006; Belis-Bergouignan et Cazals, 2006). Enfin, bien évidemment, les travaux spécifiques les plus nombreux concernent les questions de participation et de concertation (Barret, 2003; Beuret, 2006; Guilhéneuf, 2007), avec des prolongements récents sur les outils en appui à la concertation et la participation, notamment les technologies de l'information qui conduisent à revoir la notion de réseau et à s'interroger sur la construction sociale de l'information.

Au-delà des définitions et problématiques multiples à partir desquelles la gouvernance est abordée, celle-ci donne lieu aussi à des spécifications particulières en fonction des domaines, comme les notions de gouvernance européenne, urbaine, territoriale... Sans revenir sur les définitions de la gouvernance territoriale (Lardon et al., Loudiyi, dans ce numéro), notons que cette déclinaison (Bertrand et Moquay, 2004; Le Galès, 2006; Pasquier et al., 2007) s'inscrit dans l'évolution des recherches autour des territoires, comme lieux de projet collectif, d'articulation des relations global/local et de prise en compte des problématiques environnementales. Ces travaux ont permis de montrer la nécessité d'une territorialisation de la norme (Jegouzo, 2006) et l'inadaptation des territoires administratifs (Cans, 2006), conduisant à la problématique de la gouvernance multi niveaux, qui permet de contourner la question du territoire pertinent en posant celle de la coordination entre 
territoires (Valarié, 2007). La notion de gouvernance territoriale est actuellement très explorée, soit à propos de la gouvernance rurale, comme R. Welch (2002) qui confronte les théories et pratiques de gouvernement à l'échelle locale, soit à propos du développement durable, notamment la mise en œuvre des agendas 21 locaux et la concertation en appui au développement durable des territoires. Létude bibliographique du MEDD (2002) sur les territoires et le développement durable, montre que la gouvernance territoriale y occupe une place importante, avec la problématique prépondérante des jeux d'acteurs, de leurs modalités diverses de participation et de mobilisation face aux problématiques de développement durable et aux recompositions des pouvoirs. Ce type d'approche conduit à appréhender le développement durable, non pas comme « un objectif défini mais comme la formulation d'un projet par la négociation et le dialogue » (Mormont, 2001), une approche reprise par R. Laganier et al. (2002), qui évoquent un processus d'accommodement.

Au total il apparaît que les travaux consacrés aux processus et modes de gouvernance territoriale peuvent être caractérisés en croisant la nature des questions et des problématiques abordées et les méthodes et postures de recherche. On peut noter une partition entre, d'une part l'analyse ex post des dispositifs, voire l'évaluation de l'efficacité des politiques publiques, et d'autre part des travaux ciblant plutôt les représentations et le mode de construction d'une représentation ou d'un projet commun dans une logique de renforcement des processus de démocratie et d'un passage à une démocratie délibérative, ou la gouvernance en train de se faire. Cette partition recouvre, in fine, la distinction entre la gouvernance comme outil et la gouvernance comme finalité. De même, il existe un gradient dans les pratiques de recherche, qui oppose des recherches plutôt compréhensives à d'autres plus prescriptives ou impliquées, en appui à un processus de professionnalisation des savoirs mobilisés dans la mise en œuvre de la gouvernance.

\section{Éléments de gouvernance des territoires : cadrage et élargissement}

Mais finalement, c'est quoi la gouvernance territoriale? À quoi correspondent les actions de gouvernance des territoires, en particulier quand la gouvernance est appréhendée au niveau du quotidien des relations de terrain?

Tout d'abord on peut dire que la gouvernance, ce ne sont pas seulement les politiques publiques et les éléments de l'action publique. On doit en effet compter au rang des éléments de la gouvernance des territoires les lois, qui sont édictées au niveau national (code civil, code pénal, code rural, code de l'environnement...) et se déclinent aussi bien sur des territoires particuliers (lois montagne, littoral, SRU...) que sur l'ensemble des territoires institutionnels représentatifs d'une nation (loi Libertés et Responsabilités Locales...). C'est aussi le cas des règlements, qu'il s'agisse des réglementations nationales, en matière de sécurité, de social, d'étiquetage... ou des règlements et directives européens, qui proviennent de l'UE et s'appliquent de manière indifférenciée dans les différentes zones de l'Union. Mais il s'agit encore des politiques publiques, nationales ou décentralisées maintenant au niveau des grandes Régions françaises : politiques économiques, en matière de développement industriel, de services, d'agriculture ou d'énergie par exemple..., politiques sociales concernant le travail, le logement, la santé, l'éducation..., ou encore politiques d'aménagement du territoire, qu'elles soient liées à la question des infrastructures ou des dimensions foncières, si sensibles aujourd'hui. On n'oubliera pas non plus les instruments financiers (aides, taxes, impôts, contributions des usagers...), qui contribuent à orienter les politiques et les projets entrepris par les acteurs. À un niveau plus local, c'est aussi l'ensemble des documents d'urbanisme qui déterminent la manière d'habiter et d'aménager les espaces, les Plans d'occupation des sols (POS), les Plans locaux d'urbanisme (PLU), les Schémas de Cohérence Territoriale (SCoT), les schémas directeurs et plans de développement régionaux... et enfin les différents types de zonages issus de l'action publique. Les zonages territoriaux et leur diversité qui conduit aux mille feuilles des politiques et aux processus de gouvernance multi-niveaux (Pays, Communautés de Communes et d'Agglomérations, PNR, projets de territoires...), et les zonages environnementaux (Natura 
2000, Directives Oiseaux, Habitats, Znieff, corridors écologiques...), avec leurs exclusions et leurs espaces de recouvrement et de concernement complexes.

Ces éléments, qui constituent autant de dimensions de gouvernementalité des territoires, représentent une part importante de la gouvernance territoriale, sans en épuiser l'expression. Il faut en effet y ajouter les processus de coordination et les projets d'actions menés en commun par différentes catégories d'acteurs territoriaux, qu'ils soient de nature privée ou semi-publique. Distinguons ici le niveau des organisations et celui des mécanismes de gouvernance :

- au niveau des organisations, nous entendons les groupes d'acteurs qui portent les projets de territoires et structurent les relations locales, avec des niveaux variables de représentativité (Papy et Torre, 2003). Il s'agit, dans le domaine productif, des Coopératives et des Regroupements de producteurs de l'agriculture et des IAA par exemple. Ou encore des réseaux d'innovation et de transfert des technologies et de connaissances, dans le cadre des systèmes locaux de production. Citons encore les Pôles de natures diverses, sur lesquels une étude approfondie serait nécessaire, tant ils incarnent des processus et des dispositifs locaux de gouvernance territoriale : SPL, Pôles de compétitivité, PER... ou encore les Syndicats de gestion de la production (AOC) ou du territoire (Bassins versants). Dans un registre qui est davantage celui de l'aménagement du territoire et du bien commun, il faut souligner le rôle croissant joué par les Associations, qui marquent l'irruption des citoyens dans les processus de décision et la part croissante qu'ils prétendent prendre au niveau local, qu'il s'agisse de porter des projets ou de les contester. On pense en particulier aux Associations de protection de la nature, dont certaines étendent leur action au niveau national, voire au-delà, et les associations de riverains ou de voisinage, qui interviennent souvent à un niveau plus micro-local.

- Au niveau des mécanismes de gouvernance, c'est tout l'arsenal des différentes formes de participation aux débats ou à la décision qui peut être mobilisé, des formes de participation qui permettent d'associer les acteurs privés ou semi-publics aux processus de gouvernance territoriale. Les décennies précédentes ont été fertiles en inventions de toute nature visant à faciliter la mise en place du paradigme de la concertation. Citons, après J.-E. Beuret (2006), et de manière croissante selon leur intensité participative, la communication (qui a pour objet de faire passer un message et d'obtenir l'adhésion du public à une proposition), l'information (qui vise à mettre au courant un groupe des intentions ou décisions prises), la consultation (qui cherche à collecter les avis des acteurs, sans garantie quant à la prise en compte des avis exprimés), le dialogue (qui construit des interactions horizontales entre des acteurs mis sur un pied d'égalité), la concertation (qui prétend construire en commun des éléments en vue d'une solution) et enfin la négociation (qui vise à la construction commune d'une décision).

Nous avons jusqu'à présent envisagé, dans ce rapide tour d'horizon, les formes de coopération et de constructions communes au niveau local. Ce faisant, nous avons pointé la différence entre gouvernementalité et gouvernance; la gouvernance est faite de l'alliance d'acteurs de différentes catégories, de différents niveaux, qui, au-delà de leur hétérogénéité, contribuent ensemble à la définition d'actions communes et de projets collectifs. Des acteurs qui sont devenus beaucoup plus hétérogènes, en tout cas dans les zones rurales, qui se trouvent confrontés, dans leurs territoires, à des mobilités des biens et des personnes et donc des influences externes fortes, et sont sous le joug des politiques et actions publiques de toutes natures.

Toutefois, la gouvernance territoriale ne se limite pas à cette vision idyllique des relations économiques et sociales, i.e. aux formes de coopération et de constructions communes. Il s'agit également d'une interaction entre des forces poussant à la coopération, que nous venons de balayer, et d'autres forces, qui poussent au conflit. Examinons un instant les processus de développement territorial; ils ne ressemblent en aucun cas à un long fleuve tranquille. Ils sont au contraire faits de phases de négociations, de collaboration, ou d'apaisement, mais également de moments beaucoup plus animés, voire conflictuels, au cours desquels certains groupes ou certaines catégories d'acteurs s'opposent, parfois avec violence, pour définir les marches à suivre et les options à retenir. 
C'est que le processus de gouvernance des territoires présente deux faces complémentaires, dont l'importance réciproque varie selon les périodes et les situations :

- une face de nature conflictuelle;

- et une face de nature coopérative.

La compréhension de cette alchimie complexe, qui est à la base des évolutions du système local, impose la prise en considération des relations conflictuelles. Le processus de gouvernance se nourrit en effet de ces tendances opposées, conflits et coopérations, dont la synthèse conduit à la définition de sentiers de développement.

Mais pour quelles raisons les conflits occupent-ils une place dans le processus de gouvernance? Pourquoi ne pas chercher plutôt à les supprimer ou à les éradiquer? C'est que les conflits d'usage et de voisinage, et tout particulièrement les conflits d'usage de l'espace, constituent une forme de résistance, et d'expression des oppositions, à des décisions qui laissent insatisfaite une partie de la population locale. Une bonne partie des changements, des innovations locales, implique une résistance, qui peut donner lieu à la naissance de conflits. Les petits changements vont donner lieu à des tensions, ou à de petits conflits de voisinage. Les changements majeurs, qui impliquent une reconfiguration des usages de l'espace (installation d'infrastructures de transport ou de déchets, nouveaux PLUs, zonages territoriaux ou environnementaux...) donnent naissance à des conflits dont l'étendue spatiale et sociale est souvent plus importante (Torre et al., 2006). Les conflits constituent ainsi une manière d'entrer dans la discussion sur les enjeux et les chemins du développement territorial, et d'infléchir les décisions en prenant part au processus en cours alors que l'on en avait été exclu. C'est la raison pour laquelle ils portent, soit sur les décisions qui ont été prises en matière d'aménagement (négociation arbitrée), soit sur la composition et la représentativité des instances en charge de la décision (négociation arbitrale). Le conflit fait ainsi partie intégrante du processus de délibération au niveau local, en permettant une expression de la démocratie locale, ainsi que la réintégration de parties prenantes qui avaient été oubliées ou lésées dans une phase antérieure d'élaboration des projets.

Afin de comprendre la place prise par les conflits dans le processus de développement, on peut mobiliser l'analyse de A. O. Hirschman (1970, 1986), qui pose la question des réactions humaines à un choc non désiré selon le triptyque exit, voice, loyalty. Initialement élaborée pour traiter des relations de travail, cette approche peut être mobilisée comme un outil de compréhension des modes de gouvernance au niveau local, en particulier dans sa composante voice. Voici l'interprétation que nous en donnons dans le cas des processus de gouvernance des territoires (voir également Torre et Caron, 2005).

Quand se produit un événement qui laisse insatisfaite une personne (ou un groupe d'acteurs), plusieurs solutions se présentent, qui constituent autant de réponses à la situation. Précisons que le terme événement ne recouvre pas exclusivement des actes techniques avérés, tels que la construction d'un immeuble ou d'une bretelle d'autoroute, un remembrement, la pollution d'une rivière ou l'interdiction de circulation sur un espace à la propriété contestée. Il peut également s'agir d'une décision censée prendre effet dans le futur, telle que la décision de construction d'un aéroport, une enquête publique pour la construction d'installations classées, un PLU changeant la destination de certaines terres, ou encore le choix de zonages environnementaux. Les solutions issues de l'approche hirshmanienne sont au nombre de trois :

- les personnes qui considèrent que l'événement est non conforme à leurs intérêts peuvent décider de la solution d'exit. Il s'agit d'une stratégie de sortie, qui consiste, soit à quitter le territoire et à se localiser dans une zone jugée plus favorable (exit spatial, ou vote avec les pieds), soit à quitter son activité économique et à se reconvertir dans une activité compatible avec les nouvelles conditions (exit dans l'espace des ressources). On abandonne ainsi la scène de la gouvernance locale. Il va sans dire que cette solution n'est pas toujours praticable, pour des raisons financières ou de prix du foncier (par exemple, il est difficile de revendre à un bon prix des terrains pollués...). 
- Une autre solution consiste à adopter une attitude loyale à l'égard du processus en cours, c'est la loyalty. Nous l'interprétons comme l'acceptation des décisions en cours, par une participation aux dynamiques et aux projets mis en place, ainsi que par l'absence d'opposition publique. Une des manières de faire est alors d'attendre les élections suivantes, afin de présenter un programme concurrent et tenter de se faire élire.

- La troisième solution est celle du voice. Il s'agit de faire entendre sa voix et de s'opposer ainsi aux projets, aux décisions ou aux actions en cours. C'est alors la voie du conflit qui peut être choisie. Le conflit se distingue de la simple tension par le fait qu'il y a engagement des acteurs : cet engagement peut consister en des actions médiatiques, en un recours aux tribunaux, en des violences, et se manifeste souvent par la recherche d'alliés ou de partenaires de lutte. Après K. Dowding et al. (2000), on peut effectuer une distinction entre le voice individuel, plutôt réservé aux petits conflits, et le voice collectif, qui marque un élargissement de l'espace de concernement, ainsi que la mobilisation contre des projets de taille importante, ou qui impliquent une reconfiguration forte des modalités de gouvernance au niveau local. Dans ce dernier cas, le processus de voice est souvent dirigé vers les pouvoirs publics (voir Young, 1976), avec l'idée de remettre en question les décisions prises par les autorités et d'infléchir le processus de gouvernance. Le but est alors, soit de faire renoncer à de projets prévus ou déjà en cours, soit d'infléchir la décision et d'y incorporer une partie des arguments et des attentes des opposants, soit de modifier la composition des parties prenantes de la décision et d'y intégrer de nouveaux acteurs, justement ceux qui s'opposent au moyen du conflit. L'impact sur le processus de gouvernance peut être considérable : l'ampleur du conflit et la mobilisation des personnes constituent des moyens importants d’infléchissement de la décision et de prise en compte de points de vue supplémentaires conduisant à un enrichissement des processus de gouvernance des territoires à travers l'invention des nouveaux instruments, des nouveaux dispositifs.

\section{Gouvernance et situations de gestion : des outils aux dispositifs de gouvernance}

Il est à noter que la problématique de la gouvernance territoriale, conçue comme un processus qui permet à des acteurs (publics et privés) de se mettre d'accord, au sein d'un territoire donné, sur des objectifs, des actions, et des règles de coopération, recouvre largement certaines des problématiques traditionnelles des sciences de gestion. Celles-ci ont en effet élaboré une série de notions, concepts et outils méthodologiques relatifs à l'analyse des pratiques gestionnaires, concernant la conduite des organisations (Berry 1983, Moisdon 1997). On peut ainsi considérer la gouvernance comme un problème de conduite des organisations, au sens large. C'est-à-dire qu'un ensemble d'individus poursuit un ou des objectifs commun(s) et qu'ils vont mettre ensemble des moyens pour y parvenir. Dans ces situations les acteurs ont besoin, pour prendre une décision, (individuelle et collective), d'outils susceptibles de les aider dans le travail de diagnostic, d'exploration, de mise en place des actions et d'évaluation de leurs effets.

Certes, le territoire n'a jamais constitué pas un objet de recherche pour les sciences de gestion. Par exemple, E. Chia (1997) souligne que l'absence d'un responsable et/ou d'un objectif commun ne permet pas, ou rend difficile, de considérer un bassin versant comme un niveau de gestion. A. Hatchuel (2000), dans le cadre de recherches sur la RATP, note n'avoir appréhendé le territoire que pour étudier les effets de sa prise en compte dans la stratégie de l'entreprise; la notion de territoire est plus un «espace de construction de l'histoire d'une entreprise ». Plus récemment, pour N. Raulet-Crozet (2008), le territoire n'apparaît que comme « une ressource pour l'action ». Mais les situations de gouvernance territoriale se révèlent très proches de la notion de situation de gestion formalisée par J. Girin (1983) pour étudier les processus de coordination au sein des organisations, et surtout pour identifier un objet de recherche propre aux sciences de gestion. Les objectifs poursuivis peuvent aller jusqu'à (re)définir les contours du territoire, qui est dans la logique de la gouvernance une construction produite par la définition des actions à conduire. 
Pour J. Girin, « L'objet ne serait pas l'organisation, ni l'entreprise, ni même tel ou tel « morceau » d'un système socio-économique, mais quelque chose que l'on pourrait convenir d'appeler des « situations de gestion », dont l'existence autonome exigerait effectivement une approche spécifique aussi bien dans la démarche que dans les concepts ». Il formalise par la suite cette notion de situation de gestion aux situations dans lesquelles, à " un ensemble d'activités en interactions est associée l'idée d'activité collective et de résultat faisant l'objet d'un jugement », « des agents sont engagés dans la situation de gestion lorsqu'ils se reconnaissent comme participant à des degrés divers à la production du résultat».

Ainsi, dans le cas de la gestion de la forêt à Madagascar présenté par A. Toillier (ce numéro), la volonté d'élaborer et de mettre en place un nouveau mode d'exploitation de la forêt peut s'analyser en termes de situation de gestion. Celle-ci émerge en réponse à une injonction et il faut identifier les acteurs qui en font partie, les objectifs communs, les activités, etc. Le Système d'Information Géographique (SIG) apparaît alors comme un instrument, un objet intermédiaire, qui permet aux acteurs d'effectuer un état des lieux, d'explorer des mondes possibles et pourquoi pas de contrôler les actions et de faciliter l'évaluation des impacts de ces activités. Pour assurer une activité de gestion c'est-à-dire évaluer, décider et contrôler, les acteurs ont besoins d'outils, d'instruments et de dispositifs. La finalité des outils de gestion « consiste à aider un acteur ou un groupe d'acteurs à analyser les processus dans lesquels leurs actions s'inscrivent, et à anticiper leurs évolutions possibles » (Moisdon, 1997). Il apparaît que « les instruments à l'œuvre ne sont pas des dispositifs neutres, ils produisent des effets spécifiques indépendants des objectifs poursuivis et qui structurent, selon leur logique propre, l'action publique » (Lascoumes et Le Gales, 2007). Une façon d'étudier, d'analyser la situation de gestion est donc de s'intéresser aux outils, instruments et dispositifs que les acteurs utilisent pour se coordonner. C'est, par extension, la même chose pour le processus de gouvernance. Il convient donc de rappeler les principes d'étude des outils, instruments et dispositifs de gestion dont on peut s'inspirer pour les recherches sur la gouvernance.

On entend par outil de gestion «la mise en commun, l'action de relier, un certain nombre de variables qui sont des quantités, qui sont des prix, des indicateurs, des délais, etc. [...] Les outils de gestion vont donc de l'indicateur simple au modèle de la recherche opérationnelle sophistiqué » (Moisdon 1997). Les dispositifs, quant à eux, sont plus larges que les outils. Ils sont de l'ordre des arrangements entre des hommes, des objets, des règles et des outils (Moisdon, 1997). Dès le début des années 1980 les chercheurs en gestion du Centre de Recherche en Gestion (CRG) et du Centre de Gestion Scientifique (CGS) ont mis en évidence le rôle structurant des outils de gestion dans la conduite des organisations. Berry, en 1983, dans un rapport au titre évocateur, Une technologie invisible? L'impact des instruments de gestion sur l'évolution des systèmes humains, écrit que « les instruments mobilisés dans la gestion constituent un élément décisif de la structuration des situations et de leur évolution et ils engendrent souvent mécaniquement des choix et des comportements échappant aux prises des volontés des hommes, parfois même à leur conscience; ils conduisent ainsi les organisations dans des directions voulues parfois par personne et les rendent même rebelles aux efforts de réforme »(Berry, 1983). J.-C. Moisdon (1997) illustrent le rôle structurant des instruments de gestion dans des domaines aussi divers que le champagne ou la santé. Ces auteurs montrent également la diversité des formes que peuvent prendre ces instruments, plus au moins formalisés, tels que des contrats, des tableurs, des groupes de réflexion, des modèles de planification. .

À la fin des années 1990, les sociologues s’intéressent à leur tour aux dispositifs de gestion dans les organisations (Maugéri, 2001; Boussard et Maugéri, 2003; Boussard, 2008), et plus précisément aux pratiques de gestion. Maugéri (2001) définit leur problématique de recherche de la façon suivante : "Ce qui nous intéresse prioritairement, ce sont les choix d'organisation, selon une triple dimension : d'abord, à travers les principes de division et de coordination des tâches, ensuite, à travers les principes de commandement et de distribution du pouvoir, enfin, à travers les rhétoriques motivationnelles développées par le management. [...] ce que nous avons choisi d'appeler les dispositifs de gestion. » Plus récemment, dans le domaine de l'action publique, Las- 
coumes et P. Le Galès (2004) s'intéressent aux instruments de l'intervention publique. « Nous entendons par "instrumentation de l'action publique" l'ensemble des problèmes posés par le choix et l'usage des outils (des techniques, des moyens d'opérer, des dispositifs) qui permettent de matérialiser et d'opérationnaliser l'action gouvernementale. » Enfin, F. Aggeri et J. Labatut (2008) réaffirment l'intérêt des sciences de gestion à étudier l'action collective par l'analyse des outils, des instruments et des dispositifs intervenant dans les organisations. Ils évoquent l'action par les instruments : «Par ce terme, nous désignons les travaux qui proposent d'étudier l'action organisée et stratégique, non pas à travers sa substance, ses discours ou les intentions des managers, mais à travers les instrumentations, techniques, scientifiques ou gestionnaires qui sont mises en place pour conduire l'action collective et produire de nouvelles capacités. »

A. Hatchuel et B. Weil (1992) ont posé qu'une technique managériale se définit par trois éléments en interaction : un substrat technique, une philosophie gestionnaire et une vision simplifiée des relations organisationnelles. Le substrat technique est l'abstraction qui permet à un instrument de fonctionner : tableurs, ordinateurs, systèmes experts, jeux de rôle, bases de données pour des systèmes d'information géographique (SIG); alors que la philosophie gestionnaire concerne le « système de concepts qui désigne les objets et les objectifs formant les cibles d'une rationalisation » et enfin la vision simplifiée des relations organisationnelles concerne « les rôles que doivent tenir un petit nombre d'acteurs sommairement, voire caricaturalement définis » (Hatchuel et Weil, 1992).

Cette grille d'analyse peut être appliquée aux situations de gouvernance, comme en témoigne l'analyse de certains cas présentés dans ce numéro. On peut remarquer que les substrats techniques décrits ou utilisés sont multiples : depuis un jeu de cartes pour élaborer une représentation commune du territoire, jusqu'à une commission (CDOA) ou un contrat formel (CTE) ${ }^{1}$ en passant par un système de gestion de l'information au niveau global d'un pays qui mobilise à la fois des outils de planification et un cadre juridique. Il convient aussi de s'interroger sur l'avenir des instruments, qui comme dans le cas des CTE, peuvent être très contextuels et temporaires. Dès lors il convient d'étudier la genèse de ces instruments, comment ils ont été formalisés, quelles sont les controverses auxquelles ils ont donné naissance... c'est-à-dire comment ils ont pu structurer la situation de gouvernance et quels nouveaux rapports entre les acteurs locaux ils ont pu produire. Ce type de questions suppose un suivi des situations de gouvernance où un regard particulier doit être porté à l'analyse des dispositifs ou des instruments en train de se faire. C'est le cas par exemple de C. Beaurain (2008), qui en étudiant le dispositif d'écologie industrielle qui a émergé avec la création de l'association Ecopal ${ }^{2}$ en 2000, montre qu'il s'agit de configurations de coordination associant les entreprises, les collectivités locales et les acteurs institutionnels et que ce dispositif a permis de créer de nouveaux territoires. En fait ce qui est un jeu dans les situations de gouvernance c'est la production d'un langage commun, d'un projet commun, des règles qui permettent aux acteurs de mettre en place des processus d'apprentissages qui favorisent l'action commune.

1. CDOA, Commission Départementale d'Orientation Agricole. CTE, Contrat Territoriale d'Exploitation. Le CTE était un contrat signé entre les agriculteurs et l'État pour mettre en place des pratiques et systèmes de productions innovant et surtout «plus » respectueux de l'environnement. C'est la Loi d'Orientation Agricole de 1996 qui donna naissance aux CTE.

2. Ecopal: Économie et écologie Partenaires dans l'Action Locale. 


\section{Bibliographie}

Aggeri (F.), LABAtut (J.), 2008. - La gestion au prisme de ses instruments. Une analyse généalogique des approches par les instruments en gestion, Nice, Actes de la conférence AIMS, 29 p.

BARRET (Ph.), 2003. - Guide pratique du dialogue territorial. Concertation et médiation pour l'environnement et le développement local, Paris, Fondation de France, coll. «Pratiques », 136 p.

BASLÉ (M.), 2000. - «Évaluation des politiques publiques et gouvernance à différentes niveaux de gouvernement ", Cahiers Économiques de Bretagne. n² 2 p. 17-24.

Beaurain (C.), 2008. - « La construction d'un territoire à partir des ressources environnementales : l'exemple de l'agglomération dunkerquoise », Géographie Économie Société /3, Vol. 10, p. 365-384.

Belis-Bergouignan (M.-C.), Cazals (C.), 2006. - « Démarches environnementales volontaires, conflits d'usages et proximité », Revue Développement Durable et territoire, $\mathrm{n}^{\circ}$ 7, 21 p.

BerRY (M.), 1983. - Une technologie invisible? L'impact des instruments de gestion sur l'évolution des systèmes humains, Centre de recherche en gestion (CRG) de l'École Polytechnique. 60 p.

Bertrand (N.), Moquay (P.), 2004. - « La gouvernance locale, un retour à la proximité », Économie rurale $\mathrm{n}^{\circ} 280$ p. $77-95$.

Beuret (J.-E.), 2006. - La conduite de la concertation. Pour la gestion de l'environnement et la partage des ressources, Paris, L'Harmattan, $340 \mathrm{p}$.

Boussard (V.), Maugeri (S.) (dir.) 2003. - Du politique dans les organisations. Sociologies des dispositifs de gestion, Paris, L'Harmattan $280 \mathrm{p}$.

Boussard (V.), 2008. - Sociologie de la gestion. Les faiseurs de performance, Paris, Belin, 263 p.

Cans C., 2006. - «Les territoires pertinents de l'administration de l'environnement : critères et variables », dans Foucher (K.), Romi (R.) (dir.), La décentralisation de l'environnement: Territoires et gouvernance, Presses Universitaires d'Aix Marseille p. 33-47.

Capelier (W.), Simoulin (V.), 2003. - « La gouvernance : du programme de recherche à la transdisciplinarité. Présentation », Droit et Société, 54, p. 301-305.

ChiA (E.), 1997. - « Le bassin d'alimentation peut-il être un objet de gestion? Le point de vue d'un économiste ", Cahiers Agricultures, nº 6, p. 97-105.

Clark (D.), Southern (R.), Beer (J.), 2007. - « Rural governance, community empowerment and the new institutionalism: a case study of the Isle of Wight? », Journal of rural studies, 23, p. 254-266.

Denis (B.), 2008. - "L'intérêt général à l'épreuve du pluralisme », Problèmes politiques et sociaux, Paris, La Documentation française, $n^{\circ}$ 946, 118 p.

Dowding (K.), John (P.), Mergoupis (T.), Van Vugt (M.), 2000. - « Exit, voice and loyalty : Analytic and empirical developments », European Journal of Political Research, 37, p. 469-495.

Ehlinger (S.), Perret (V.), Chabaud (D.), 2007. - «Quelle gouvernance pour les réseaux territorialisés d'organisations? », Revue française de gestion, n 170, p. 155-171.

Hatchuel (A.), Weil (B.), 1992. - L'expert et le système, Paris, Economica, 263 p.

Gaudin (J.-P.), 2002. - Pourquoi la gouvernance?, Paris, Presses de Sciences Po, coll. « La bibliothèque du citoyen », $138 \mathrm{p}$.

—, 1999. - Gouverner par contrat. L'action publique en question. Presses de Sciences Po, Paris, 233 p.

Guilhéneuf (P.-Y.), (dir.) 2007. - Concertation et territoires. Quels dispositifs, quels enjeux?, Mémoire du Séminaire du 21 avril 2007, Paris, Geyser, 34 p.

Girin (J.), 1983. - «Les situations de gestion », dans BERRY (M.) (dir.), Le rôle des outils de gestion dans l'évolution des systèmes sociaux complexes, CRG-École Polytechnique, Rapport pour le ministère de la Recherche et de la Technologie, 5 p.

HATChuel (A.), 2000. - Recherche, intervention et production des connaissances, Montpellier, Séminaire INRA Pour et sur le développement, 12 janvier 2000,14 p.

Heurgon (E.), Landrieu (J.), 2000. - Prospective pour une gouvernance participative. Colloque de Cerisy, La Tour-d'Aigues, Éditions de L'aube, coll. «L'aube territoire. Série Prospective du présent », 380 p.

Hirschman (A. O.), 1970. - Exit, voice and loyalty: Responses to decline in firms, organizations and states, Cambridge, M.A., Harvard University Press, 162 p. 
—, 1986. - Exit and voice. An expanding sphere of influence, dans Hirschman (A. O.) (dir.), Rivals views of market society and other essays, Harmondsworth, Penguin, p. 77-101.

Hufty (M.), Dormeier Freire (A.), Plagnat (P.), Neumann (V.), 2007. - Jeux de gouvernance regards et réflexions sur un concept, Paris, IUED/Karthala, coll. « Développement », $242 \mathrm{p}$.

JÉGOUZO (Y.), 2006. - « Existe-t-il un territoire pertinent? », dans Foucher (K.), Romi (R.) (dir.), La décentralisation de l'environnement : territoires et gouvernance, Presses Universitaires d'Aix Marseille, p. 121126.

KeEfer (P.), 2004. - A review of the political economy of governance: from property rights to voice. Work bank Policy Research Working paper 3315, may 2004, 49 p.

Laganier (R.), Villalba (B.), Zuindeau (B.), 2002. - « Le développement durable face au territoire : éléments pour une recherche interdisciplinaire », Développement Durable et Territoire, Dossier n ${ }^{\circ} 1$ : Approches territoriales du développement durable, 26 p.

LAHAYE (N.), 1999. - Gouvernance territoriale et espaces d'intérêt publics : l'enjeu d'un développement durable territorial. Communication à l'école chercheur économie spatiale et régionale 8-10 décembre 1999 Le Croisic, $24 \mathrm{p}$.

LARRUe (C.), 2000. - Analyser les politiques publiques d'environnement, Paris, L'Harmattan, coll. « Logiques politiques », $207 \mathrm{p}$.

Lascoumes (P.), 2005. - « Le développement durable : vecteur d'innovations politiques ? », dans Le développement durable. Les termes du débat, Paris, Armand Colin, p. 95-107.

Lascoumes (P.), Le Gales (P.), (dir.), 2004. - Gouverner par les instruments, Paris, Sciences Po, 370 p.

Lascoumes (P.), Le Galès (P.), 2007. - Sociologie de l'action publique, Paris, Armand Colin, 128 p.

LE GALÈs (P.), 2006. - Gouvernement et gouvernance des territoires. Problèmes politiques et sociaux. n 922 mars 2006, La Documentation Française Ed., Paris, 119 p.

—, 1995. - «Du gouvernement des villes à la gouvernance urbaine », Revue française de Science Politique, 45(1) p. 27-95.

Maugéri (S.), (dir.), 2001. - Délit de gestion, Paris, La dispute, 246 p.

Meisel (N.), Ould Aoudia (J.), 2007. - La bonne gouvernance est-elle une bonne stratégie de développement? Document de travail Agence Française de Coopération, 69 p.

MEDD, 2002. - Étude bibliographique sur les territoires et le développement durable. Rapport final, Paris, Ministère de l'Écologie et du Développement Durable et fondation des villes, Direction des études économiques et de l'évaluation environnementale, service de la recherche et de la prospective, 163 p + annexes.

Moisdon (J.C.), (dir.), 1997. - Du mode d'existence des outils de gestion - les instruments de gestion à l'épreuve de l'organisation, Éditions Seli Arslan, 286 p.

Mormont (M.), 2001. - «Gouverner l'environnement », dans DebuYst (F.) et al., Savoirs et jeux d'acteurs pour des développements durables, Louvain-la-Neuve, Bruylant-Academia, p. 221-236.

Ost (F.), 2004. - «De la pyramide au réseau : un nouveau paradigme pour la science du Droit? », dans Supiot (A.), Tisser le lien social (dir.), Paris, Maison de la Science de l'Homme, p. 175-196.

Papy (F.), TorRe (A.), 2003. - «Quelles organisations territoriales pour concilier production agricole et gestion des ressources naturelles? ", Études et Recherches sur les Systèmes Agraires et le Développement, $n^{\circ} 33$, p. 151-169.

Pasquier (R.), Simoulin (V.), Weisbein (J.), 2007. - La gouvernance territoriale. Pratiques, discours et théories. Droit et Société, vol. 44, Paris, LGDJ, 234 p.

Platteau (J.-P.), 2003. - Droits de propriété et gestion efficace des ressources naturelles. Les séminaires de l'IDDRI n 10, juillet 2003, $39 \mathrm{p}$.

Raulet-Croset (N.), 2008. - «Action publique et territoires locaux : la gestion de situations "intermédiaires" », RFG/4, n 184, p. 151-165.

Rey-Valette (H.), LaRdon (S.), Chia (E.), 2008. - « Governance: Institutional and learning plans facilitating the appropriation of sustainable development ", International Journal of Sustainable Development, Volume 11 ( $2 \& 3), 10 \mathrm{p}$. 
Salles (D.), 2006. - Les défis de l'environnement. Démocratie et efficacité, Paris, Syllepse, coll. « Ecologie et Politique », $250 \mathrm{p}$.

TAiclet (A.F.), 2007. - « Le développement économique territorial au regard des hypothèses de la gouvernance territoriale », dans Pasquier (R.), Simoulin (V.) Weisbein (J.) (dir.), La gouvernance territoriale. Pratiques, discours et théories, Paris, LGDJ, coll. « Droit et Société; 44 », p. 109-127.

TALвот (D.), 2006. - « La gouvernance locale, une forme de développement local et durable ? Une illustration par les pays », Développement durable et Territoires, Dossier n ${ }^{\circ} 7$ : «Proximité et environnement », $24 \mathrm{p}$.

Torre (A.), Aznar (O.), Bonin (M.), Caron (A.), Chia (E.), Galman (M.), Guérin (M.), Jeanneaux (P.), Kirat (T.), Lefranc (C.), Melot (R.), Paoli (J.-C.), Salazar (M.-I.), Thinon (P.), 2006. - « Conflits et tensions autour des usages de l'espace dans les territoires ruraux et périurbains. Le cas de six zones géographiques françaises ", Revue d'Economie Régionale et Urbaine, n³, p. 415-453.

Torre (A.), Zuindeau (B.), 2006. - Éditorial « Proximité et Environnement », Développement Durable et Territoires, Dossier 7 : « Proximité et Environnement », 8 p.

Torre (A.), Caron (A.), 2005. - « Réflexions sur les dimensions négatives de la proximité : le cas des conflits d'usage et de voisinage », Économie et Institutions, n 6-7, p. 183-220.

VALARIÉ (P.), 2007. - L'eau à l'épreuve de la biodiversité: la gouvernance multiniveaux, Communication au Joint Congress of the European Regional Science Association et Association de Sciences Régionale de Langue française, Paris 29 août, 2 septembre, Session spéciale Concepts et outils de gouvernance territoriale, $14 \mathrm{p}$.

WeLCH (R.), 2002. - « Legitimacy of rural local government in the new governance environment », Journal of rural studies, 18, p. 443-459.

Young (D. R.), 1976. - « Consolidation or diversity: choices in the structure of urban governance », American Economic Review, 66, p. 378-385.

Zaoual (H.), 2005. - Socio économie de la proximité. Du local au global, Paris, L'Harmattan, coll. « Économie plurielle», $189 \mathrm{p}$. 